\title{
Geotechnical Engineering: Referees 2011
}

The following is a list of referees who have reviewed papers for Geotechnical Engineering between 1 January 2011 and 31 December 2011. The Institution of Civil Engineers is very grateful for their assistance.

We are continually looking for suitable reviewers for papers submitted to Geotechnical Engineering. Papers published in the Proceedings of the ICE must be submitted to at least two independent referees to judge accuracy, style, impact, importance and interest.

If you are interested in reviewing articles on any topic related to

E. Akcali

H. Amick

C. Anagnostopoulos

M. Arnold

A. Arulrajah

A. Asadi

J. H. Atkinson

M. Attom

C. Augarde

B. Avar

R. Bailey

G. Barnes

F. Basile

D. Baxter

D. Beadman

L. Begaj

S. Bird

J. Black

M. Boutouil

A. Brennan

A. Brown

D. Bruce

G. Bunce

H. J. Burd

S. Buttling

B. Byrne

J. Castro

H. Y. Chai

A. Chan

T. Chapman

Q. Chen

E. L. Chin

A. Chodorowsk

J. Chu

S. Clarke

J. Claydon

J. Colmenares

N. Consoli

M. Coop

J. Cripps

M. Czerewko

L. Di Matteo

M. Diakoum

M. Dobie
G. Dounias

T. Dyer

R. Eduardo

D. Egan

A. El Nahas

G. Elia

G. Elliott

C. Erbrich

A. Eslami

C. Evans

R. J. Fannin

E. Farrell

R. Fell

P. Fleming

J. Gannon

K. Gardiner

K. Gavin

G. Ghatoaora

P. Gilbert

A. Gillarduzzi

C. Golightly

D. Greenwood

M. Griffith

W. Guo

C. Harikumar

J. Harrison

A. Holeyman

V. Hope

A. Hosseinzadeh

Y. S. Hsu

E. Ibraim

G. Iskander

P. Jamsawang

R. Jardine

D. Jarman

A. Javadi

M. Jefferies

D. S. Jeng

B. Jones

C. Jones

C. J. F. P. Jones

M. Kahyaoglu

M. Kamiura

T. Koumoto geotechnical engineering, please submit your name, qualifications or CV, and areas of expertise. We are in need of individuals who will agree to review papers in a timely fashion (within 3 to 4 weeks of receipt) and provide confidential feedback to the Editorial Advisory Panel concerning the quality of the paper and any suggested revisions that would be appropriate.

If you are such a person, please contact Sarah Walker (tel.: +44 (0)207 665 2450; e-mail: sarah.walker@ice.org.uk) for more information on the referee process.

P. V. Lade

K. Law

Y. R. Li

M. Lings

J. P. Love

A. Lutenegger

M. Maugeri

P. Mayne

M. McCarthy

R. Meriggi

C. Merrifield

A. Merritt

G. Milligan

F. Milne

A. Modaressi

F. Molenkamp

J. Monnet

D. Mothersille

A. Muir Wood

D. Muir Wood

W. Murphy

S. A. Naeini

P. Naughton

R. Neilson

M. Nguyen

K. Nicholls

D. Nicholson

P. Nowak

M. O'Neill

S. Olivella

J. Omer

T. Orr

A. Ortigao

A. Osman

F. Ostadan

D. Page

E. Passaris

D. M. Potts

H. G. Poulos

J. Powell

W. Prakoso

M. C. Preziosi

M. Redaelli

S. Reid

T. Roberts

C. Robinson

A. Rodger

A. Rose

P. Rutty

R. Salgado

V. Saouma

J. Sharma

G. Shore

S. K. Shukla

B. Simpson

V. Sivakumar

H. Skinner

D. Smith

M. Smith

P. Smith

J. Standing

G. Swift

M. A. Syed

N. Tafazzol

G. Taylor

B. Teymur

R. Thompson

A. Tolooiyan

E. Uygar

V. van Beek

S. Vanapall

C. Viggiani

H. Villar

H. Vrecl-Kojc

P. Waddell

S. Walthall

N. Wharmby

M. Whitbread

J. Yang

T. Y. Yap

Y. Yilmaz

Q. Yue

L. Zdravkovic

L. Zhang

M. E. Raghunandan

C. Raison 